\title{
Germinação de sementes e emergência de plântulas de carapiá: espécie primitiva e medicinal
}

\author{
José Magno Q Luz'; André F Carvalho'; ${ }^{1}$ Denise G de Santana ${ }^{1}$; Monalisa AD Silva ${ }^{2}$ \\ ${ }^{1}$ UFU-ICA, Bloco 2E, Campus Umuarama, 38400-902 Uberlândia-MG; ${ }^{2} U F R P E-U A S T, ~ 56900-000$ Serra Talhada-PE; jmagno@, \\ umuarama.ufu.br; monallyysa@yahoo.com.br
}

\section{RESUMO}

Dorstenia cayapia Vellozo (Moraceae), popularmente conhecida por carapiá, é uma espécie primitiva do ponto de vista geológico e ao mesmo tempo evoluída do ponto de vista de estruturas morfológicas; com amplo uso medicinal é pouco estudada quanto à sua capacidade reprodutiva. Diante disso, e pelo fato de Dorstenia cayapia ser uma espécie ameaçada de extinção, o objetivo deste trabalho foi determinar o potencial da espécie para propagação por sementes por meio de seus atributos biológicos e tecnológicos. O experimento foi instalado em delineamento inteiramente casualizado em esquema fatorial 2 x 3 sendo o primeiro fator correspondente à temperatura (constante em $25^{\circ} \mathrm{C}$ e alternada $20-30^{\circ} \mathrm{C}$ ) e o segundo fator ao substrato (vermiculita, papel mata-borrão e papel de filtro), com quatro repetições de 20 sementes por parcela. $\mathrm{O}$ uso dos substratos vermiculita e papel mata-borão à temperatura de $25^{\circ} \mathrm{C}$ aumentaram o percentual de plântulas normais e de sementes germinadas. A germinação das sementes tornou-se mais lenta quando as sementes foram dispostas em papel de filtro, assim como se tornaram mais assíncronas nesse substrato. A temperatura alternada $20-30^{\circ} \mathrm{C}$ atrasou o início do processo de germinação das sementes de carapiá.

Palavras-chave: Dorstenia cayapia, espécie medicinal, germinação de sementes.

\begin{abstract}
Seed germination and seedling emergence of Dorstenia cayapia: primitive and medicinal species

Dorstenia cayapia Vellozo (Moraceae), popularly known as carapiá, is a primitive species from the geological point of view but well evolved from the point of view of morphological structures; of large medicinal use, this plant reproductive capacity was not well studied. Since this species is threatened with extinction, the objective of this study was to evaluate the physiological quality of its seeds by their biological and technological attributes. The experiment was set up in a completely randomized design with a factorial $2 \times 3$ scheme, in which the first factor was the temperature (constant of $25^{\circ} \mathrm{C}$ and alternated $20-30^{\circ} \mathrm{C}$ ) and the second factor was the substrate (vermiculite, rolled paper and filter paper), with four replications of 20 seeds per plot. The use of the substrates vermiculite and rolled paper at $25^{\circ} \mathrm{C}$ increased the percentage of normal seedlings and germinated seeds. The seeds germination was slower when they were placed on filter paper, and it was more asynchronous in this substrate. Additionally, the alternating temperature $20-30^{\circ} \mathrm{C}$ delayed the beginning of the germination of Dorstenia cayapia seeds.
\end{abstract}

Keywords: Dorstenia cayapia, medicinal species, seeds germination.

(Recebido para publicação em 27 de abril de 2009; aceito em 1 de fevereiro de 2010) (Received on April 27, 2009; accepted on February 1, 2010)

A família Moraceae apresenta aproximadamente 61 gêneros, com mais de 1000 espécies frequentes nas regiões tropicais e subtropicais de todo o mundo e menos comuns em climas temperados (Cronquist, 1981; Joly, 1991). Da família, o gênero Dorstenia está dividido em nove seções baseadas no habitat e nas características morfológicas (Berg \& Hijman, 1999) e tem se distinguido dos demais pela predominância de espécies herbáceas (Berg, 2001; Zerega et al., 2005).

As espécies de Dorstenia teriam surgido no Cretáceo há cerca de 100 milhões de anos, quando a América do Sul e a África estavam parcialmente unidas (Carauta \& Valente, 1974; McLoughin, 2001). É um gênero primitivo, sob o ponto de vista da geologia histórica e, ao mesmo tempo, evoluído considerando- se as estruturas morfológicas, como nervação, adaptação e resistência à seca e polinização entomófila (Carauta, 1978). O gênero tem distribuição pantropical, com 37 espécies no Brasil (Carauta, 1978; Carauta \& Valente, 1983; Carauta \& Albuquerque, 2002). São mais conhecidas popularmente por carapiás, embora sejam também nominadas de caiapiá, contra-erva, tiú, figueirilha, chupa-chupa, liga-liga, capa-homem, conta-de-cobra, sabugo-roxo, liga-osso, figueirinha, figueira-da-terra, dentre outros (Carauta, 1978; Lifchitz, 1981).

Em condições ecológicas favoráveis, os carapiás florescem e frutificam todo ano (Carauta, 1978) e têm preferência por sub-bosques sombreados e úmidos das florestas tropicais (Araújo et al., 2007). Os frutos são do tipo drupa (Granville, 1971), mas pouco se conhece sobre o papel do exocarpo na dispersão das espécies de carapiás (Kaufmann et $a l ., 1991)$, embora se saiba que, quando maduro, exerce pressão e ejeta o endocarpo contendo a semente (Berg, 1977).

São numerosos os trabalhos científicos publicados sobre a composição química e a ação medicinal dos carapiás, principalmente pela ação das furanocumarinas em doenças de pele (Celeghini et al., 2007), alguns de etnobotânica (Rodrigues \& Carvalho, 2001; Guarim Neto \& Morais, 2003; Oliveira et al., 2003), apenas um de micropropagação com uma espécie africana, Dorstenia gigas (Krogstrup et al., 2005) e nenhum sobre as formas de propagação dos carapiás brasileiros.

Diante disso, e pelo fato de carapiá ser uma espécie ameaçada de extin- 
ção (Fahrig, 2003), o objetivo deste trabalho foi determinar o potencial da espécie para propagação por sementes por meio de seus atributos biológicos e tecnológicos.

\section{MATERIAL E MÉTODOS}

De 400 plantas de carapiá localizadas no município de Raul Soares-MG, foram coletados em 2007, 21 cenantos femininos (receptáculo carnoso). Desses, os frutos foram extraídos (1159 frutos), contando-se o número médio de sementes viáveis por cenanto e o número de frutos (drupas) por cenanto. Após secagem à sombra e com a pressão dos dedos foram eliminados os frutos chochos e imaturos, restando os inteiros, que serão referenciados como sementes, ainda que sejam uma drupa.

Para determinação do teor de água, as sementes foram colocadas em estufa à temperatura de $105 \pm 3^{\circ} \mathrm{C}$ por 24 horas a partir de duas subamostras de 20 sementes. A determinação da massa de mil sementes foi feita conforme prescrições incluídas nas Regras para Análise de Sementes (Brasil, 1992).

No teste de germinação, sementes de carapiá foram dispostas segundo delineamento experimental inteiramente casualizado em esquema fatorial $2 \times 3$ sendo o primeiro fator correspondente à temperatura (constante em $25^{\circ} \mathrm{C}$ e alternada $\left.20-30^{\circ} \mathrm{C}\right)$ e o segundo fator ao substrato (vermiculita, papel mataborrão e papel de filtro), com quatro repetições de 20 sementes por parcela. $\mathrm{O}$ recipiente utilizado com vermiculita e papel mata-borão foi caixa de plástico $(11,0 \times 11 \times 3,5 \mathrm{~cm})$, enquanto que, com papel de filtro, o recipiente foi a placa de Petri.

Para testar o efeito temperatura, câmaras de germinação foram ajustadas e equipadas com lâmpadas fluorescentes brancas, com irradiância de $440 \mathrm{~mW}$ $\mathrm{cm}^{-2}$ e fotoperíodo de $12 \mathrm{~h}$ de luz e 12 $\mathrm{h}$ de escuro. Os substratos, previamente esterilizados, foram umedecidos com água desionizada na proporção de 2,5 vezes a massa do substrato seco. Aos 44 dias após a instalação, o teste foi encerrado quando verificado que as sementes ainda não germinadas mostraram-se visivelmente deterioradas.

Dois critérios distintos foram aplicados na avaliação do processo, o de plântulas normais, critério agronômico (Brasil, 1992) e o de protrusão radicular ou percentual de germinação, critério fisiológico. Ainda, baseado no critério fisiológico, foram calculados os tempos inicial, médio e final de germinação, velocidade de emergência e sincronia. As expressões e autores dessas medidas podem ser consultados em Ranal \& Santana (2006).

Todas as características foram inicialmente testadas quanto às pressuposições de normalidade dos resíduos (teste de Shapiro-Wilk) e homogeneidade entre as variâncias (teste de Bartlett). Como para todas as características as pressuposições foram atendidas, transformações de dados não foram utilizadas e por isso a análise de variância para o modelo fatorial foi aplicada aos dados originais. Em função dos fatores temperatura e substrato serem qualitativos do ponto de vista estatístico, as comparações das médias foram feitas pelo teste de Tukey a $\alpha=0,05$ de significância. Para o estudo do comportamento da semente ao longo do período de germinação, gráficos de distribuição de frequência foram construídos para cada temperatura e substrato.

\section{RESULTADOS E DISCUSSÃO}

As 400 plantas de carapiá analisadas, produziram em média 55 cenantos, sendo o número de frutos por cenanto extremamente variável, entre 10 a 94 , com média de 55 frutos por cenanto. Esse valor foi muito próximo à média de 61 frutos por cenanto obtida para plantas de Dorstenia bonijesu cultivadas em casa de vegetação (Araújo et al., 2007). Apesar das espécies de Dorstenia apresentarem grande produção de frutos, esta característica, segundo Venable (1997), não é medida confiável do sucesso reprodutivo. Ainda segundo o autor, a avaliação de sucesso deverá levar em conta, além do percentual de germinação, a porcentagem de plântulas normais. $\mathrm{O}$ peso de mil sementes estimado foi de 1,724 g, com teor de água, em média, de $6,5 \%$.
De maneira geral, as sementes foram mais rápidas para iniciar o processo de germinação $\left(t_{\mathrm{o}}\right)$ em temperatura constante de $25^{\circ} \mathrm{C}$, embora quando semeadas sobre papel mata-borão tenham sido indiferentes à temperatura (Tabela 1). As sementes demoraram mais para iniciar a germinação (23 dias) quando dispostas em papel de filtro e em temperatura alternada. Quanto ao tempo médio, as sementes foram indiferentes à temperatura, com exceção da semeadura sobre papel de filtro que além de aumentar o tempo médio em relação aos demais substratos, exigiu menor tempo médio em temperatura alternada $20-30^{\circ} \mathrm{C}$.

As temperaturas e os substratos utilizados não afetaram o tempo final de germinação das sementes, que variou entre 28 e 30 dias (Tabela 2). A temperatura constante de $25^{\circ} \mathrm{C}$ estimulou de forma semelhante o percentual de germinação das sementes (critério fisiológico) e de plântulas normais, em relação à temperatura alternada (Tabela 1). Em revisão sobre a temperatura ótima para a germinação de sementes de espécies da família Moraceae, Brancalion et al. (2007) encontraram temperaturas constantes de $25^{\circ} \mathrm{C}$ para Brosimum gaudichaudii, 25,30 e $35^{\circ} \mathrm{C}$ para Brosimum rubescens, $30^{\circ} \mathrm{C}$ para Clarisia racemosa, 25 e $30^{\circ} \mathrm{C}$ para Helicostylis tomentosa e 20,25 e $30^{\circ} \mathrm{C}$ para Maclura tinctoria. Isso indica que espécies da família não respondem à alternância de temperatura. Soma-se a isso, o fato de D. cayapia, habitar locais sombreados (Araújo et al., 2007), ou mesmo microhabitats ciliares como Dorstenia asarioides (Lima et al., 2007), onde possivelmente as oscilações de temperatura sejam mínimas. Ausência de plântulas normais em temperaturas alternadas (Tabela 2) reforça essa constatação.

Valores de velocidade de emergência $(V E)$ abaixo de 1 , indicam que em média o número de plântulas por dia é baixo e por vezes nenhuma germinação é observada (Tabela 2). Como reflexo, a sincronia $(Z)$ de germinação das sementes é baixa. A falta de sincronia na germinação parece ser característica comum entre espécies florestais, sejam elas arbóreas, sub-arbóreas ou arbustivas, para evitar que toda a produção de sementes fique concentrada num curto 
Tabela 1. Tempos inicial e médio de germinação de sementes de carapiá (initial and average time to the beginning of carapiá seeds germination). Uberlândia, UFU, 2008.

\begin{tabular}{|c|c|c|c|c|c|c|}
\hline \multirow[t]{2}{*}{ Substrato } & \multicolumn{3}{|c|}{$\begin{array}{c}t_{0}(\mathrm{dia}) \\
\text { Temperatura }\left({ }^{\circ} \mathrm{C}\right)\end{array}$} & \multicolumn{3}{|c|}{$\begin{array}{c}\bar{t}(\mathbf{d i a}) \\
\text { Temperatura }\left({ }^{\circ} \mathrm{C}\right)\end{array}$} \\
\hline & 25 & $20-30$ & média & 25 & $20-30$ & média \\
\hline Vermiculita & $14,2 \mathrm{aA}$ & $17,2 \mathrm{aB}$ & 15,7 & $20,2 \mathrm{aA}$ & $19,8 \mathrm{aA}$ & 20,0 \\
\hline Papel mata-borão & $14,8 \mathrm{aA}$ & $15,8 \mathrm{aA}$ & 15,3 & $21,4 \mathrm{aA}$ & $19,5 \mathrm{aA}$ & 20,5 \\
\hline Papel de filtro & $15,8 \mathrm{aB}$ & $22,0 \mathrm{bA}$ & 18,9 & $25,7 \mathrm{bB}$ & $20,6 \mathrm{aA}$ & \\
\hline média & 14,9 & 18,3 & & 22,4 & 20,0 & \\
\hline$D M S_{\text {temperatura }}$ & \multicolumn{3}{|c|}{2,47} & \multicolumn{2}{|c|}{0,98} & \\
\hline$D M S_{\text {substrato }}$ & \multicolumn{2}{|c|}{2,99} & & \multicolumn{2}{|c|}{3,48} & \\
\hline
\end{tabular}

$t_{\mathrm{o}}=$ tempo inicial de germinação; $\bar{t}=$ tempo médio de germinação $\left(t_{\mathrm{o}}=\right.$ initial time to beginning of germination; $\bar{t}=$ average time to beginning of germination).

Tabela 2. Medidas de germinação e de plântulas normais de carapiá (Dorstenia cayapia) submetidas a diferentes substratos e temperaturas de incubação (germination periods of carapiá seeds in relation to different substrates and incubation temperatures). Uberlândia, UFU, 2008.

\begin{tabular}{lccccc}
\hline Temperatura $\left({ }^{\circ} \mathbf{C}\right)$ & $\boldsymbol{t}_{\mathrm{f}}(\mathbf{d i a})$ & $\boldsymbol{G} \mathbf{( \% )}$ & $\boldsymbol{P ( \% )}$ & $\begin{array}{c}\boldsymbol{V} \boldsymbol{( \%} \\
\left(\mathbf{p l a ̂ n t . ~ d i a}^{-1}\right)\end{array}$ & $\boldsymbol{Z}$ \\
\hline 25 & $29,60 \mathrm{a}$ & $42,33 \mathrm{a}$ & $41,60 \mathrm{a}$ & $0,459 \mathrm{a}$ & $0,080 \mathrm{a}$ \\
$20-30$ & $27,93 \mathrm{a}$ & $31,67 \mathrm{~b}$ & $0,00 \mathrm{~b}$ & $0,308 \mathrm{~b}$ & $0,108 \mathrm{a}$ \\
\hline$D M S_{\text {temperatura }}$ & 4,17 & 8,15 & 9,23 & 0,083 & 0,055 \\
\hline Substrato & & & & \\
\hline Vermiculita & $29,10 \mathrm{a}$ & $42,50 \mathrm{a}$ & $25,00 \mathrm{a}$ & $0,453 \mathrm{a}$ & $0,148 \mathrm{a}$ \\
Papel mata-borão & $29,10 \mathrm{a}$ & $52,00 \mathrm{a}$ & $37,00 \mathrm{a}$ & $0,536 \mathrm{a}$ & $0,103 \mathrm{ab}$ \\
Papel de filtro & $28,10 \mathrm{a}$ & $16,50 \mathrm{~b}$ & $0,00 \mathrm{~b}$ & $0,159 \mathrm{~b}$ & $0,032 \mathrm{~b}$ \\
\hline$D M S_{\text {substrato }}$ & 6,19 & 12,09 & 18,21 & 0,123 & 0,082 \\
\hline$t_{\mathrm{f}=;} G=; P=; V E=; Z=$ sincronia de germinação & &
\end{tabular}

período de tempo. Apesar das dorstênias serem de um gênero de predominância de espécies herbáceas, o gênero evolui de uma família (Moraceae) de gêneros em sua totalidade arbóreos ou arbustivos, como o Ficus, Morus, Artocarpus, Brosimum e Cecropia (Barth, 1976).

Fatores genéticos e ambientais podem afetar a uniformidade e a sincronia de germinação. A protoginia, que é processo de amadurecimento dos frutos a partir de flores pistiladas antes das estaminadas, comuns em espécies de Dorstenia (Carauta, 1978; Berg, 2001), podem produzir sementes com graus diferentes de maturação e consequentemente irregularidade no processo de germinação.

Com exceção do tempo final, o uso de papel de filtro reduziu a capacidade $\left(20-30^{\circ} \mathrm{C}\right)$, principalmente para a vermiculita e papel de filtro, com tendência da germinação ficar concentrada próxima ao pico. Sob temperaturas constantes, embora não apresentassem altos picos, a germinação se prolongou ao longo do tempo de observação.

As frequências com vários picos, independente da temperatura e do substrato, indicam distribuição não normal às sementes de carapiá. Essa característica implica em germinação incerta (Labouriau \& Valadares, 1976; Labouriau, 1983), típica de espécies não melhoradas. Este comportamento pode ser importante para a manutenção de suas sementes do banco no solo (Garwood, 1989).

\section{REFERÊNCIAS}

ARAÚJO LM; VIEIRA MF; GODOY AG. 2007. Biologia reprodutiva de Dorstenia binijesu MORACEAE. Série Urticineae 28:198-204.

BARTH OM. 1976. Catálogo sistemático dos polens das plantas arbóreas do Brasil Meridional. XXIII. Moraceae. Memórias do Instituto Oswaldo Cruz 74: 295-309.

BERG CC. 1977. Urticales, their differentiation and systematic position. Plant Systematics and Evolution 1: 349-374.

BERG CC; HIJMAN MEE. 1999. The genus Dorstenia (Moraceae). Ilicifolia 2: 1-211.

BERG CC. 2001. Moraceae, Artocarpeae, and Dorstenia (Moraceae) - with introduction to the family and Ficus and with additions and corrections to Flora Neotropica. New York: The New York Botanical Garden. 347p.

BRANCALION PHS; NOVEMBRE ADLC; RODRIGUES RR; CHAMMA HMCP. 2007. Estabelecimento da temperatura ótima para a germinação das sementes de 272 espécies arbóreas nativas do Brasil. Informativo Abrates 17: 55-68.

BRASIL Ministério da Agricultura, Pecuária e Abastecimento. 1992. Regras para análise de sementes. Brasília: SNAD/DNDV/CLAV. $365 \mathrm{p}$.

CARAUTA JPP; VALENTE MC. 1974. Dorstenia L. (Moraceae) dos estados da Guanabara e do Rio de Janeiro. Rodriguésia 39: 225-296.

CARAUTA JPP. 1978. Dorstenia L. (Moraceae) do Brasil e países limítrofes. Rodriguésia 44:53-223.

CARAUTA JPP; VALENTE MC. 1983. Dorstenia (Moraceae). Notas complementares IV. Atas da Sociedade Botânica do Brasil 1:111-122.

CARAUTA JPP; ALBUQUERQUE JM. 2002. Dorstenia (Moraceae). Notas complementares V. Albertoa 8: 53-56.

CELEGHINI RMS; YARIWAKE JH; LANÇAS FM. 2007. Otimização das condições de extração hidroalcoólica das furanocumarinas de Dorstenia brasiliensis Lam. por maceração 


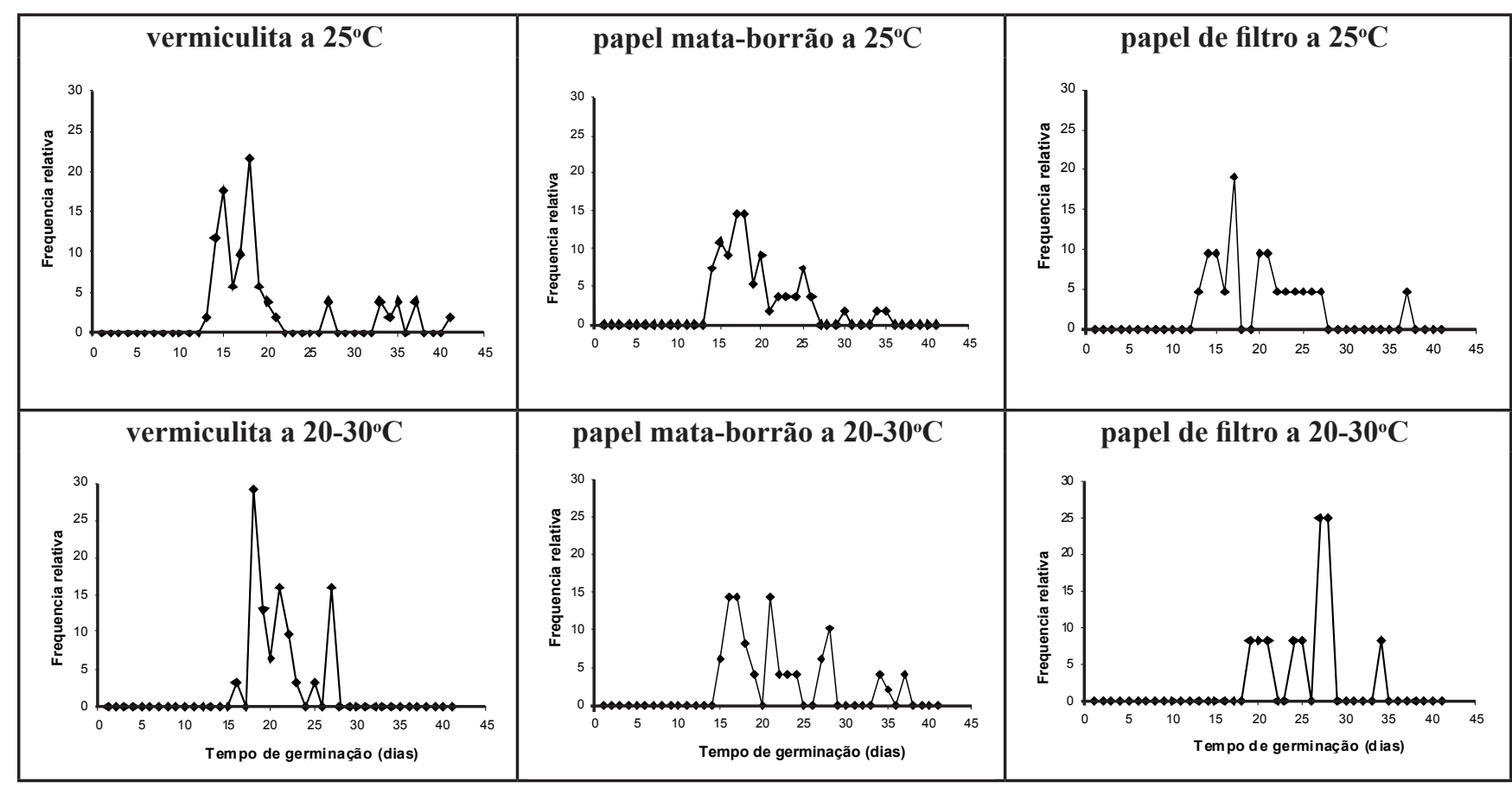

Figura 1. Distribuições de frequência relativa à germinação de sementes de carapiá (Dorstenia cayapia) submetidas a diferentes substratos para semeadura (vermiculita, papel mata-borão, papel de filtro) e temperaturas de incubação $\left(25^{\circ} \mathrm{C}\right.$ e alternada $\left.20-30^{\circ} \mathrm{C}\right)($ Frequency distribution from the germination of carapiá seeds submitted to various substrata(vermiculite, rolled paper and filter paper)). Uberlândia, UFU, 2008.

com ultra-som e análise quantitativa por CLAE/UV e fluorescência. Revista Brasileira de Plantas Medicinais 9: 61-66.

CRONQUIST A. 1981. An integrated system of classification of flowering plants. New York: Columbia University. 1262p.

FAHRIG L. 2003. Effects of habitat fragmentation on biodiversity. Annual Review of Ecology and Systematics 34: 487-515.

GARWOOD NC. 1989. Tropical soil seed banks: a review. In: LECK MA; PARKER VT; SIMPSON RL (eds). Ecology of soil seed banks. London: Academic Press. p. 149-209.

GRANVILLE JJ. 1971. Notes sur la biologie florale de qulques espèces du genre Dorstenia (Moracées). Cahiers ORSTOM. Série Biologie 15: 61-97.

GUARIM NETO G; MORAIS RG. 2003. Recursos medicinais de espécies do Cerrado de Mato Grosso: um estudo bibliográfico. Acta Botanica Brasilica. 17: 561-594.

JOLY AB. 1991. Botânica: introdução à taxonomia vegetal. 2 ed. São Paulo: Editora Nacional, Ed. Universidade de São Paulo, 777p.

KAUFMAN S; MCKEY DB; HOSSAERTMCKEY M; HORVITZ CC. 1991. Adaptations for a two-phase seed dispersal system involving vertebrates and ants in a hemiepiphytic fig (Ficus microcarpa: Moraceae). American Journal of Botany 78: 971-977.

KROGSTRUP P; FIND JI; GURSKOV DJ; KRISTENSEN MMH. 2005. Micropropagation of Socotran fig, Dorstenia gigas. Scrweinf. In vitro cellular \& Developmental Biology-Plant 41: 81-86.

LABOURIAU LG. 1983. A germinação das sementes, Monografias Científicas, Washington, USA, $170 \mathrm{p}$.

LABOURIAU LG; VALADARES MB. 1976. On the germination of seeds of Calotropis procera. Anais da Academia Brasileira de Ciências 48:174-186.

LIFCHITZ A. 1981. Plantas medicinales. 5. ed. Buenos Aires: Kier. 139p.

LIMA EM; ARAÚJO EL; SAMPAIO EVS; FERRAZ EMN; SILVA KA; PIMENTEL RMM. 2007. Fenologia e dinâmica de duas populações herbáceas da caatinga. Revista de Geografia 24: 120-136.

MCLOUGHLIN S. 2001. The breakup history of Gondwana and its impact on pre-Cenozoic Xoristic provincialism. Australian Journal of
Botany 49: 271-300

OLIVEIRA FQ; JUNQUEIRA RG; STEHMANN JR; BRANDÃO MGL. 2003. Potencial das plantas medicinais como fonte de novos antimaláricos: espécies indicadas na bibliografia etnomédica brasileira. Revista Brasileira de Plantas Medicinais 5: 23-31.

RANAL MA; SANTANA DG. 2006. How and why to measure the germination process? Revista Brasileira de Botânica 29: 1-11.

RODRIGUES VEG; CARVALHO, DA. 2001. Levantamento etnobotânico de plantas medicinais no domínio do cerrado na região do Alto Rio Grande - Minas Gerais. Ciência e Agrotecnologia 25: 102-123.

VENABLE DL. 1997. Packaging and provisioning in plant reproduction. In: SILVERTOWN M; FRANCO M; HARPER JL (Eds.). Plant Life Histories. Ecology, Phylogeny and Evolution. Cambridge: Cambridge University Press. p. 163-190.

ZEREGA NJC; CLEMENT, WL; DATWYLER SL; WEIBLEN GD. 2005. Biogeography and divergence times in the mulberry family (Moraceae). Molecular Phylogenetics and Evolution 37: 402-416. 